\title{
SINTOMAS DEPRESSIVOS E USO DE CANNABIS EM ADOLESCENTES
}

\author{
Tânia Moraes Ramos Andrade* \\ Irani Iracema de Lima Argimon ${ }^{\#}$
}

\begin{abstract}
RESUMO. A depressão é um dos transtornos psiquiátricos mais comuns na adolescência. Os quadros depressivos costumam apresentar elevadas taxas de comorbidades psiquiátricas, sendo freqüente o abuso de substâncias psicoativas. O artigo investiga a associação dos sintomas depressivos e o uso da cannabis. Método: Revisão sistemática, análise dos artigos indexados no Medline, PsycInfo, ProQuest, Web of Science e Lilacs, entre 2000 e 2005, descritores: depressive symptoms, depressive, adolescence, teenager e cannabis. Resultados: Revisados 36 artigos completos, resultando no estudo de 9 artigos, que tratam de sintomas depressivos ou depressão e o uso ou dependência de cannabis em adolescentes. Os estudos confirmam a associação entre sintomas depressivos e o uso de cannabis na adolescência, sendo esta associação mais freqüente no uso precoce e regular de cannabis. Conclusão: Os sintomas depressivos/depressão estão relacionados ao uso/abuso e dependência de cannabis na adolescência. A investigação clínica e os programas de prevenção devem abordar estes transtornos na adolescência.
\end{abstract}

Palavras-chave: depressão, adolescência, cannabis.

\section{DEPRESSIVE SYMPTOMS IN YOUNG CANNABIS USERS}

\begin{abstract}
The association between depressive symptoms and cannabis use is investigated. Due to the fact that depression has been a common psyquiatric disorder in adolescence, depressive cases usually present high rates of psychiatric comorbidities and the abuse of psychoactive substances is frequent. Systematic review, studies at PsycInfo, ProQuest, Web of Science and Lilacs databases analyzed between 2000 and 2005 are discussed. Results show that nine out of the thirty-six studies discuss depression and cannabis use or addiction in adolescents. Whereas the association between depressive symptoms and the use of cannabis during adolescence is confirmed, it is more frequent in an early and continuous cannabis use. Research shows that depressive symptoms are related to cannabis use, abuse and dependence during adolescence. Abovementioned variables must be investigated in clinical practice and in prevention programs that simultaneously focus on these disorders.
\end{abstract}

Key words: Depression, adolescence, cannabis.

\section{SÍNTOMAS DEPRESIVOS EN ADOLESCENTES USUARIOS DE CANNABIS}

RESUMEN. Este artículo busca investigar la asociación de los síntomas depresivos y el uso de cannabis en la adolescencia. Método: Ha sido realizado, a través de revisión sistemática, el análisis de los artículos indexados localizados en los sistemas Medline, PsycInfo, ProQuest, Web of Science y Lilacs, entre 2000 y 2005, utilizando los descriptores: depressive symptoms, depressive, adolescence, teenager y cannabis. Resultados: La mayoría de los estudios confirma existir una asociación entre síntomas depresivos y el uso de cannabis en la adolescencia, cabe destacar que esta asociación es más frecuente en el uso precoz y regular de cañabais. Conclusión: Los síntomas depresivos/Depresión están relacionados al uso/abuso y dependencia de cañabais en la adolescencia, siendo entonces importante que estas variables puedan ser investigadas en la práctica clínica como también en programas de prevención que aborden de forma simultánea estos síntomas/trastornos en este período de la vida.

Palabras-clave: Depresion; adolescencia; cannabis.

A adolescência é caracterizada por intensas transformações tanto físicas quanto psicológicas.
Embora ocorram mudanças fisiológicas em todas as fases do ciclo vital, a rapidez das transformações

* Mestre em Psicologia Clínica. Docente da Faculdade de Psicologia da Universidade Luterana do Brasil-ULBRA/Canoas.

\# Doutora em Psicologia Clínica. Docente Titular do Programa de Pós-Graduação da Pontifícia Universidade Católica do Rio Grande do Sul. 
durante o período da adolescência é maior do que nos anos que a precedem. Esta fase do desenvolvimento tem a duração de quase uma década, indo dos $12 \mathrm{ou}$ 13 anos até em torno dos 20 anos (Papalia \& Olds, 2000).

Bessa (2004) refere que a adolescência é marcada por transformações tanto orgânicas como emocionais, sendo um período propício ao surgimento de transtornos psiquiátricos.

Entre os transtornos psiquiátricos mais comuns na adolescência, a depressão é considerada debilitante e recorrente e envolve um alto grau de morbidade e mortalidade, representando um sério problema de saúde pública. De 15 a $25 \%$ das pessoas podem apresentar crise depressiva pelo menos uma vez na vida, sendo que o primeiro episódio depressivo ocorre, mais freqüentemente, antes dos dezoito anos de idade (Bahls \& Bahls, 2002).

No Brasil, nos últimos anos tem-se observado interesse maior em verificar a prevalência de sintomas depressivos entre os adolescentes. Salle (1999), em estudo com 503 estudantes do Ensino Médio de Porto Alegre, da faixa de 15 a 17 anos de idade, identificou a prevalência de $32 \%$ de sintomas depressivos, sendo que $5 \%$ da amostra apresentaram Depressão Maior. Gorenstein, Andrade, Zanolo e Artes (2005) identificaram, em uma amostra de 1.555 adolescentes de 13 a 17 anos de idade, que $7 \%$ destes adolescentes apresentavam depressão. $\mathrm{O}$ instrumento utilizado neste estudo para investigar sintomas depressivos foi $o$ Inventário Beck de Depressão (Steer \& Beck, 1996), sendo o escore acima de 20 pontos. As meninas, nesta amostra, apresentaram mais sintomas depressivos do que os meninos. Os autores também encontraram um aumento dos escores de sintomas depressivos nos adolescentes com mais idade.

Outro problema que afeta os adolescentes que apresentam quadros depressivos é a elevada ocorrência de co-morbidade, isto é, duas entidades diagnósticas em um mesmo indivíduo (Laranjeira, Zaleski \& Ratto, 2004), sendo freqüente o abuso e a dependência de substâncias psicoativas com os quadros de depressão (Bahls \& Bahls, 2002; Bessa, 2004; Friedberg \& McClure, 2004; Sukiennik \& Salle, 2002).

Segundo o V Levantamento Nacional Sobre Consumo de Drogas Psicotrópicas entre Estudantes do Ensino Fundamental e Médio da Rede pública de Ensino nas 27 Capitais Brasileiras (Galduróz, Noto, Fonseca \& Carlini, 2005) Porto Alegre foi a capital que mais apresentou uso de cannabis $(10,9 \%)$.

$\mathrm{O}$ uso de cannabis pelos adolescentes, principalmente o uso regular e pesado, tem sido associado com a depressão por muitos pesquisadores, como Degenhardt, Hall e Lynskey (2003), Fergusson, Howood e Swain-Campell (2002), Raphael, Wooding, Stevens e Connor (2005), Rey, Martin e Krabman (2004) e Saluja et al. (2004). Cabe salientar que cada estudo utiliza diferentes critérios para classificação quanto à frequiência de uso de cannabis, assim como Fergusson et al. (2002), que classificam a frequiência de uso anual de cannabis pelos adolescentes em nãouso de cannabis, uso de menos que uma vez por mês (1-11), uso de pelo menos de 12 a 50 vezes ao mês e uso de mais que 50 vezes ao mês. Já Rey et al. (2004) utilizam os critérios de freqüência de uso de cannabis como: uso regular mais que 5 vezes durante o mês e o uso pesado como mais de 40 vezes ao mês. Salienta-se que, quanto à frequiência do uso de cannabis, nos estudos estas variam entre as classificações de nãouso, uso regular e uso pesado de cannabis.

Tendo em vista a prevalência de sintomas depressivos e os quadros de depressão entre os adolescentes e a possível associação deste transtorno psiquiátrico com o uso, abuso ou dependência de cannabis, este estudo tem por objetivo identificar as publicações existentes nas bases de dados computadorizadas escolhidas no período de 2000 a 2005, através do método de revisão sistemática relacionada com esta temática.

\section{MÉTODO}

O método utilizado foi a busca sistemática através das bases de dados computadorizadas Medline, PsycInfo, ProQuest e Web of Science no período compreendido entre 2000 e 2005, com os seguintes descritores: depression, depressive, adolescence, teenager e cannabis.

Foram incluídos artigos empíricos em língua inglesa das bases referidas, com amostras de adolescentes, não importando seu delineamento. As referências dos estudos identificados também foram utilizadas. Os estudos selecionados deveriam abordar a associação entre sintomas depressivos ou depressão e uso, abuso ou dependência de cannabis. Foram excluídos os artigos em outras línguas que não o Inglês, revisões da literatura e pesquisas cujo objetivo fosse enfocar: prevalência, fatores de risco e tratamento.

Nesta busca, foram identificadas 80 referências, cujos resumos foram checados em busca de artigos potencialmente elegíveis, resultando em 36 artigos completos que foram avaliados de acordo com os critérios estabelecidos, e destes restaram 9 artigos que investigaram a associação de sintomas depressivos ou depressão e uso, abuso ou dependência de cannabis, 
os quais foram incluídos neste estudo. O critério utilizado para esta seleção foi que os estudos contemplassem a associação de sintomas depressivos/Depressão, cannabis e adolescência.

\section{RESULTADOS}

Os 9 artigos obtidos por meio da revisão sistemática apresentaram dados em relação à base populacional, ao número de participantes, ao delineamento e à classificação quanto ao uso, abuso ou dependência de cannabis e sintomas depressivos ou depressão maior e critérios diagnósticos utilizados no estudo para a avaliação de sintomas depressivos, depressão maior, uso, abuso ou dependência de cannabis. Esses dados se encontram resumidos na Tabela 1.

Tabela 1. Características dos Estudos Envolvendo o Uso de Cannabis e Sintomas Depressivos em Adolescentes

\begin{tabular}{|c|c|c|c|c|c|c|c|}
\hline Autor & $\begin{array}{l}\text { Tipo de } \\
\text { Estudo }\end{array}$ & $\mathbf{n}$ & Base & Cannabis & DP & $\begin{array}{c}\text { Critérios } \\
\text { Diagnóstico } \\
\text { cannabis } \\
\end{array}$ & $\begin{array}{c}\text { Critérios } \\
\text { Diagnóstico } \\
\text { Depressão }\end{array}$ \\
\hline Boys et al., 2003 & TV & 2.624 & $\mathrm{BP}$ & $\mathrm{UC}$ & DP & Questionário Freq./Uso & $\begin{array}{c}\text { CIDI } \\
\text { DSM-IV }\end{array}$ \\
\hline Blore et al., 2004 & $\mathrm{CO}$ & 975 & $\mathrm{BP}$ & DC & DP & $\begin{array}{l}\text { Questionário } \\
\text { Freq./Uso }\end{array}$ & $\begin{array}{l}\text { Questionário } \\
\text { desenvol. } \\
\text { autores }\end{array}$ \\
\hline Brook et al., 2002 & $\mathrm{CO}$ & 975 & BP & DC & DP & $\begin{array}{c}\text { DMS-IV } \\
\text { Questionário } \\
\text { Freq./Uso }\end{array}$ & DSM-IV \\
\hline Chabrol et al., 2005 & TV & 212 & $\mathrm{BP}$ & DC & DP & $\begin{array}{l}\text { DSM-IV } \\
\text { Questionário } \\
\text { Freq/Uso } \\
\text { MINI }\end{array}$ & CES-D \\
\hline Fergusson et al., 2002 & $\mathrm{CO}$ & 1.265 & BA & DC & DP & $\begin{array}{l}\text { Questionário } \\
\text { Freq./Uso }\end{array}$ & $\begin{array}{c}\text { DSM-IV } \\
\text { CIDI }\end{array}$ \\
\hline Libby et al., 2005 & $\mathrm{CO}$ & 126 & $\mathrm{BA}$ & DC & DP & $\begin{array}{l}\text { CIDI-SAM } \\
\text { DISC-IV } \\
\text { DSM-IV }\end{array}$ & $\begin{array}{l}\text { DSM-IV } \\
\text { CDRS-R }\end{array}$ \\
\hline Patton et al., 2002 & $\mathrm{CO}$ & 1.601 & $\mathrm{BP}$ & DC & DP & $\begin{array}{l}\text { Questionário } \\
\text { Freq./Uso }\end{array}$ & CISR-R \\
\hline Poulin et al., 2005 & TV & 12.771 & $\mathrm{BP}$ & $\mathrm{UC}$ & $\mathrm{SD}$ & $\begin{array}{c}\text { Questionário } \\
\text { Freq./Uso } \\
\text { Westminster } \\
\text { Drugs } \\
\text { and Alcohol Questionnaire }\end{array}$ & $\begin{array}{l}\text { CES-D } \\
\text { The Moods and Feelings Questionnaire } \\
\text { (MFQ) } \\
\text { K-SADS DSM-IV }\end{array}$ \\
\hline
\end{tabular}

Legenda: BP - base populacional; BA - base ambulatorial; CO - coorte; TV - transversal; UC - uso de cannabis; AC - abuso de cannabis; DC - dependência de cannabis; SD - sintomas depressivos; DP - depressão

Poulin, Hand, Boudreau e Santor (2005) investigaram adolescentes escolares canadenses e verificaram que há diferença na prevalência de sintomas depressivos de acordo com o gênero. Nas meninas a prevalência de sintomas depressivos foi de $8,6 \%$, enquanto nos meninos foi de $2,6 \%$ e, no que diz respeito ao uso da cannabis (diário ou semanal), no último mês, foi encontrado que este uso, independentemente da sua quantidade, era indicador de sintomas depressivos em ambos os sexos.

Yates, Kramer e Garralda (2004) investigaram sintomas depressivos em adolescentes que foram atendidos em ambulatório de clínica geral com idades de 13 a 16 anos. Estes autores constataram que maior grau de sintomas depressivos estava associado com alguns fatores de risco tais como: gênero (feminino), sintomas psiquiátricos parentais, sintomas físicos, prejuízos psicossociais, ter um amigo usuário de drogas, ter oferta de drogas por meio de um amigo ou estranho no início do uso, usar cannabis e uso diário de tabaco. A conclusão dos autores foi que o uso de cannabis estava associado com o grau de sintomas depressivos, porém não deixa claro se este uso referese ao uso na vida ou uso atual, nem sua freqüência. 
Cabe salientar que nos estudos de Poulin et al. (2005) e Yates et al. (2004) foram investigados sintomas depressivos e o uso de cannabis.

Em estudo com 212 adolescentes com idades entre 15 e 22 anos, Chabrol, Ducongé, Casas, Roura e Carey (2005) constataram que 54\% dos adolescentes usaram cannabis nos últimos 6 meses, entre estes, $45 \%$ eram meninas, com média de idade de 18,9 anos, e $55 \%$ eram meninos, com média de idade de 18,6 anos. Os meninos iniciaram o uso, em média, com 14 anos e as meninas, em média, com 16 anos. A duração do uso de cannabis foi maior nos meninos (média de 3,9 anos) do que nas meninas (média de 3 anos). Entre os que referiram uso de cannabis, $64 \%$ dos meninos e $34 \%$ das meninas preenchiam critérios de dependência para com esta classe de substância. Não foi encontrado, neste estudo, associação entre sintomas depressivos ou de ansiedade com a motivação para o uso de cannabis.

Pesquisadores como Boys et al. (2003), tendo investigado adolescentes com 13 a 15 anos de idade, encontraram associação entre tabagismo, uso regular (definido pelos autores como mais de uma vez por mês) de álcool e de cannabis com um aumento de 4 a 5 vezes na incidência de Transtorno Depressivo.

Libby, Orton, Stover e Riggs (2005) compararam uma amostra de adolescentes com diagnóstico de Depressão Maior com um grupo de adolescentes que apresentava transtorno por uso de substâncias psicoativas (isoladamente ou em co-morbidade com a depressão), encontrando uma maior prevalência de dependência da cannabis nos adolescentes diagnosticados com Depressão Maior antes do uso da substância. Entre os que apresentaram Depressão primária, a dependência de cannabis foi o transtorno por uso de substância mais prevalente entre os meninos, enquanto a dependência de tabaco foi o de maior prevalência nas meninas.

Brook, Brook, Zhang, Cohen e Whiteman (2002) concluíram que existe uma forte associação entre a frequiência do uso de cannabis em adolescentes e Depressão Maior antes dos 20 anos de idade e que o uso precoce de substâncias psicoativas está associado à presença de transtornos psiquiátricos.

Pesquisando associação entre depressão, uso de cannabis e comportamento suicida em adolescentes, com idades variando entre 14 e 21 anos, Fergusson et al. (2002) concluíram que existe uma associação entre o uso semanal de cannabis e depressão, ideação suicida e tentativa de suicídio. Os adolescentes de 14 a 15 anos de idade que usaram cannabis semanalmente apresentaram maiores taxas de ideação suicida do que os não-usuários e do que os adolescentes usuários de 20 à 21 anos.

Blore, Schulze e Lessing (2004) realizaram pesquisa com adolescentes de 13 a 17 anos. Em relação à prevalência de sintomas depressivos, as meninas apresentaram um índice mais elevado de sintomas depressivos do que os meninos. Os autores obtiveram resultados que mostraram uma correlação positiva entre sintomas depressivos e a precocidade e frequiência do uso de substâncias psicoativas, entre elas a cannabis, no último mês, não sendo encontrada diferença entre o uso de substâncias psicoativas e o gênero. Os fatores de risco identificados para depressão e uso de substâncias psicoativas incluem: conflito no relacionamento com os pais, experiência de eventos estressantes, insatisfação com as notas escolares e ter amigos que são usuários de substâncias psicoativas. Os sentimentos de agressão, raiva, incapacidade e culpa foram observados por metade da amostra, enquanto ideação suicida foi referida por 75\% dos adolescentes.

Em estudo de seguimento de 07 anos, Patton, Coffey, Degenhhardt, Lynskey e Hall (2002) investigaram 1.601 adolescentes de 14 a 15 anos e concluíram que $60 \%$ dos adolescentes já haviam usado cannabis até a idade de 20 anos. Destes adolescentes, $7 \%$ usaram cannabis diariamente. Os adolescentes que faziam uso semanal ou mais freqüente de cannabis tinham duas vezes mais risco de apresentarem, posteriormente, diagnósticos de depressão e ansiedade. Por outro lado, o diagnóstico de depressão e ansiedade não foi indício de uso diário ou semanal de cannabis, o que contraria a hipótese de que adolescentes depressivos utilizam cannabis como automedicação.

\section{DISCUSSÃO}

É importante salientar que, mesmo sendo bastante difundida a alta prevalência de sintomas depressivos na adolescência, é encontrado um número reduzido de estudos (Blore et al., 2004; Boys et al., 2003, Brook et al., 2002; Chabrol et al., 2005; Fergusson et al., 2002; Libby et al., 2005; Patton et al., 2002; Poulin et al., 2005; Yates et al., 2004) que avaliaram sintomas depressivos/depressão e uso/abuso ou dependência de cannabis em adolescentes.

O predomínio dos estudos desta revisão foi de publicações dos anos de 2004 e 2005, de estudos com delineamento de coorte, de base populacional, e estudos que apresentaram investigação de diagnóstico de dependência de cannabis e de Depressão Maior. 
Destaca-se, na análise dos resultados obtidos, a alta prevalência dos sintomas depressivos e uso de cannabis em adolescentes (Chabrol et al., 2005; Poulin et al., 2005; Yates et al., 2004), o que demonstra a importância do estudo destas questões quando se planeja implementar programas direcionados a esta clientela que visem à prevenção primária, secundária e terciária.

Os sintomas depressivos são mais freqüentes e intensos no sexo feminino, conforme os achados de vários pesquisadores (Poulin et al., 2005; Yates et al., 2004), o que aponta a necessidade de que haja respeito às especificidades de cada sexo ao serem delineadas abordagens terapêuticas para adolescentes.

Nota-se que nem todos os estudos pesquisaram dependentes ou abusadores de cannabis, atendo-se ao uso desta substância (Blore et al., 2004; Yates et al., 2004), assim como nem todos diagnosticaram depressão, investigando apenas os sintomas depressivos (Blore et al., 2004; Chabrol et al., 2005; Poulin et al., 2005; Yates et al., 2004). O uso de cannabis, nestes estudos, pode significar desde um uso na vida (experimental) até uma Síndrome de Dependência, com toda a sua complexidade e custos associados, tanto pessoais quanto sociais, familiares e ocupacionais. Da mesma forma, os sintomas depressivos podem ser analisados em um continuиm que vai desde sintomas de intensidade leve até um quadro clínico de Depressão Maior. É importante que estas variáveis sejam bem definidas nos estudos para possibilitar uma análise consistente de seus resultados. Este cuidado pôde ser observado nos estudos analisados (Boys et al., 2003; Brook et al., 2002; Fergusson et al., 2002; Libby et al., 2005), os quais se preocuparam em delimitar os critérios diagnósticos e os instrumentos utilizados para tal, havendo uma única exceção (Yates et al. 2004), em que não fica claro se o uso de substâncias psicoativas refere-se a uso na vida ou uso atual, o que, em termos de pesquisa e da clínica, faz uma enorme diferença.

Alguns pesquisadores, como Poulin et al. (2005) e Yates et al. (2004), verificaram uma maior prevalência de sintomas depressivos em adolescentes que fazem uso de cannabis. Em outros estudos identificou-se que os adolescentes dependentes de cannabis eram mais propensos a apresentar depressão (Boys et al., 2003; Brook et al., 2002; Libby et al., 2005; Patton et al., 2002), ocorrendo também uma associação entre uso de cannabis e Depressão Maior (Boys et al., 2003; Brook et al., 2002; Fergusson et al., 2002; Patton et al., 2002). Uma questão a ser debatida é a relação temporal entre a dependência de cannabis (ou seu uso) e a Depressão Maior (ou sintomas depressivos). Foi encontrado, em estudo conduzido por Libby et al. (2005), que os participantes com história prévia de Depressão Maior apresentaram mais dependência de cannabis do que aqueles sem esta história, o que aponta para a presença de uma co-morbidade psiquiátrica. Deve-se ressaltar, no entanto, que tanto a intoxicação quanto os sintomas de abstinência ou um transtorno induzido pelo uso da cannabis devem ser considerados quando se abordam sintomas depressivos em usuários desta substância psicoativa (Organização Mundial da Saúde, 1993).

A precocidade do uso de cannabis, esteve associada tanto aos sintomas depressivos (Boys et al., 2003; Blore et al., 2004; Poulin et al., 2005, Yates et al., 2004) quanto à Depressão Maior (Brook et al., 2002; Patton et al., 2002), o que sugere a importância da detecção precoce deste uso no intuito de prevenir o desenvolvimento destes quadros clínicos. O uso regular de cannabis, assim como sua precocidade, também esteve associado à ideação e tentativas de suicídio, como relatam Fergusson et al. (2002), o que pode ser utilizado como um argumento contra aqueles que consideram inócuo o uso da cannabis, difundindo esta idéia nos meios de comunicação.

A associação entre depressão/sintomas depressivos e uso/abuso ou dependência de cannabis leva a questionar quanto o tratamento da Depressão Maior pode ter um impacto na redução do uso da cannabis assim como referem Chabrol et al. (2005) e Patton et al. (2002). Não é por acaso que o tratamento sinérgico da dependência e da depressão é a abordagem preconizada, como salientam Laranjeira et al. (2004), quando se trata destes transtornos, devendo o profissional que atende este tipo de paciente acompanhar com atenção as características específicas de cada quadro.

\section{CONCLUSÕES}

As publicações indexadas nesses últimos anos evidenciaram que os estudos têm avançado na investigação de diferentes aspectos que podem interferir no desenvolvimento normal do adolescente.

A depressão na adolescência gera enormes dificuldades tanto emocionais quanto sociais e intelectuais. O sofrimento causado pela depressão faz com que o jovem muitas vezes procure saídas rápidas, como o uso de substâncias psicoativas ou até mesmo o suicídio. Da mesma forma, verifica-se que o uso de substâncias psicoativas, entre elas a cannabis, também vem acompanhado de uma série de dificuldades e sintomas, podendo ser destacados os sintomas depressivos. 
Nos estudos investigados evidenciou-se maior predominância de sintomas de depressivos nas meninas do que nos meninos. $\mathrm{O}$ uso precoce, regular e pesado de cannabis esteve associado com sintomas depressivos e depressão. A existência da associação entre sintomas depressivos/depressão e uso/abuso ou dependência de cannabis em adolescentes foi marcante em todos os estudos.

O conhecimento da existência da associação entre sintomas depressivos/depressão e uso/abuso ou dependência de cannabis pode contribuir na implantação de programas voltados para os adolescentes, visando à prevenção em seus três níveis - o primário, o secundário e o terciário -, sendo importante que os profissionais que trabalham com adolescentes tenham clara a possibilidade da existência de co-morbidade entre depressão e dependência de cannabis. As áreas da pesquisa e da clínica devem, assim, ser analisadas de forma integrada para um delineamento de estratégias terapêuticas mais efetivas no atendimento mais integrado do adolescente.

Sugere-se para os novos estudos a utilização de outros termos descritivos, como marijuana, no intuito de abranger um número maior de experimentos.

\section{REFERÊNCIAS}

Bahls, S. \& Bahls, F. (2002). Depressão na adolescência: características clínicas. Interação em Psicologia, 6(1),49-57.

Bessa, M. (2004). Quando o uso de drogas ocorre junto com outros transtornos psiquiátricos. Em L. Pinsky \& M. A. Bessa (Orgs.), Adolescência e drogas (pp. 124-150). São Paulo: Contexto.

Blore, L., Schulze, S. \& Lessing, A. (2004). The relationship between adolescent depressive symptomology and substance abuse. Curationis, 27(1), 12-22.

Boys, A., Farrell, M., Taylor, C., Marsden, J., Goodman, R., Brugha, T., Bebbington, P., Jenkins, R. \& Meltzer, H. (2003). Psychiatric morbidity and substance use in young people aged 13-15 years: Results from the Child and Adolescent Survey of Mental Health. British Journal of Psychiatry, 182(6), 509-517.

Brook, D., Brook, J., Zhang, C., Cohen, P. \& Whiteman, M. (2002). Drug use and risk of major depressive disorders, alcohol dependence, and substance use disorders. Archives of General Psychiatry, 59(11), 1039-1044.

Chabrol, H., Ducongé, E., Casas, C., Roura, C. \& Carey, K. (2005). Relations between cannabis use and anxious, depressive and borderline symptomatology. Addiction Behavior, 30(4), 829840.

Degenhardt, L., Hall, W. \& Lynskey, M. (2003). Exploring the association between cannabis use and depression. Addiction, 98(11), 1493-1504.

Fergusson, D., Howood, J. \& Swain-Campell, N. (2002). Cannabis use and psychosocial adjustment in adolescent and young adulthood. Addiction, 97(9), 1123-1135.
Friedberg, R. \& McClure, J. (2004). Trabalhando com crianças e adolescentes deprimidos. Em R. Friedberg \& J. McClure (Orgs.), A prática clínica de terapia cognitiva com crianças e adolescentes (pp. 146 -172). Porto Alegre: Artmed.

Galduróz, J., Noto, A., Fonseca, A. \& Carlini, E. (2005). V Levantamento Nacional sobre o consumo de drogas psicotrópicas entre estudantes do ensino fundamental e médio da rede pública de ensino nas 27 capitais brasileiras 2004. São Paulo: UNIFESP.

Gorenstein, C., Andrade, L., Zanolo, E. \& Artes, R. (2005). Expression of depressive symptoms in a nonclinical Brazilian adolescent sample. Cannadian Journal of Psychiatry, 50(3), 129-136.

Laranjeira, R., Zaleski, M. \& Ratto, L. (2004). Comorbidades psiquiátricas: uma visão global. Em ABEAD (Org.), Comorbidades: transtornos mentais $x$ transtornos por uso de substancias de uso (pp. 5-9). São Paulo: ABEAD.

Libby, A., Orton, H., Stover, S. \& Riggs, P. (2005). What came first, major depression or substance use disorder? Clinical characteristics and substance use comparing teens in a treatment cohort. Addiction Behavior, 30(9), 1649-1662.

Organização Mundial de Saúde (1993). Classificação de Transtornos Mentais e de Comportamento da CID-10: descrições clínicas e diretrizes diagnósticas. Porto Alegre: Artes Médicas.

Papalia, D. \& Olds, S. (2000). Desenvolvimento físico e cognitivo. Em D. Papalia \& S. Olds (Orgs.), Desenvolvimento Humano (7 ed., pp. 310-311). Porto Alegre: Artmed.

Patton, G., Coffey, C., Degenhardt, L., Lynskey, M. \& Hall, W. (2002). Cannabis use and mental health in young people: Cohort study. British Medical Journal, 325(7374), 1195-1198.

Poulin, C., Hand, D., Boudreau, B. \& Santor, D. (2005). Gender differences in the association between substance use and elevate depressive symptoms in a general adolescent population. Addiction, 100(4), 525-535.

Raphael, B., Wooding, S., Stevens, G. \& Connor, J. (2005). Comorbidity: Cannabis and complexity. Psychiatric Practice, 11(3), 161-176.

Rey, J., Martin, A. \& Krabman, P. (2004). Is the party over? Cannabis and juvenile psychiatric disorder: The past 10 years. Journal of American Child Adolescent Psychiatry, 43(10), 1194-1205.

Salle, E. (1999). Estudo de sintomatologia depressiva em adolescentes de 15 a 17 anos de II grau de Porto Alegre, através das escalas auto-aplicáveis Beck Depression Inventory (BDI) Carrol Rating Scale (CRS), Center for Epidemiological Studies for depression (CES-D). Dissertação de Mestrado não publicada, Programa de Pós-Graduação em Clínica Médica, Universidade Federal do Rio Grande do Sul, Porto Alegre.

Saluja, G., Iachan, R., Scheidt, P., Overpeck, M., Sun, W. \& Giedd, J. (2004). Prevalence of and risk factors for depressive symptoms among young adolescents. Archives of Pediatric \& Adolescent Medicine, 158(8), 760-765.

Steer, R. \& Beck, A. (1996). Beck Depression Inventory (BDI). In L. I. Sederer \& B. Dickey (Eds.), Outcomes assessment in clinical practice (pp. 100-104). Baltimore: Williams \& Wilkins.

Sukiennik, P. \& Salle, E. (2002). Depressão. Em R. Souza \& M. Costa (Orgs.), Adolescência aspectos clínicos e psicossociais (pp. 340-349). Porto Alegre: Artmed. 
Yates, P., Kramer, T. \& Garralda, E. (2004). Depressive symptoms

Recebido em 08/12/2006 amongst adolescent primary care attenders. Levels and

Aceito em 13/06/2007 associations. Social Psychiatry and Psychiatric Epidemiology, 39(7), 588-594.

Endereço para correspondência : Tânia Moraes Ramos Andrade. Rua Florêncio Ygartua, 288 conj. 303, Moinhos de Vento, CEP 90430-010, Porto Alegre-RS E-mail: taniaandrade@terra.com.br 